\title{
Análise quantitativa e qualitativa do nível de conhecimento dos alunos do curso de fisioterapia sobre a atuação do fisioterapeuta em saúde pública
}

\author{
Quantitative and qualitative analysis of the level of knowledge \\ from the physiotherapy student's course, \\ about the performance of the physiotherapist in public health
}

Cristiane Roberta $\mathrm{N}$ aves ${ }^{1}$ Vanessa de Souza Brick ${ }^{2}$

${ }^{1}$ Universidade Estadual Paulista Júlio de M esquita Filho. Distrito de Rubião Júnior s/n. 18618-970 Botucatu SP. crica_naves@hotmail.com ${ }^{2}$ Faculdade $M$ arechal Rondon.
Abstract Physiotherapy is the science of health that studies, prevents and takes care of the functional kinetic disturbsin essential organs and system of human body. The objective was to examine the expectation of students at $1^{\text {st }}, 3^{\text {rd }}$ and $5^{\text {th }}$ semester of physiotherapy course about the physiotherapist activity in public health and the expectancy on the Family Health Program (PSF). The study took place in $\mathrm{M}$ arechal Rondon College, with 107 students, having as exclusion criterion the students from the $7^{\text {th }}$ semester. A questionnaire with 11 questions was used being, eight multiple option questions and three discursive questions. For the statistic analysis, it was used the descriptive method with M icrosoft Excel 2003 Program and speech's analysis of the collective subject. In relation to the physiotherapist actuation in the U nified Health System (SU S) $44 \%$ of students think it is very important, $36 \%$ think that the physiotherapist function in the PSF is very important whereas $24 \%$ of students thinks don't know about the efficiency of its attendance. The students think that the physiotherapist in the SU S and PSF isimportant, but a little of them have knowledge about the physiotherapist performance in Public Health.

Key words Physiotherapy, Public health, National H ealth System
Resumo Fisioterapia é a ciência da saúde que estuda, previne etrata os distúrbios cinéticos funcionais em órgãos e sistemas do corpo humano. 0 objetivo deste estudo é verificar a expectativa dos alunos do primeiro ao quinto semestre de fisioterapia sobre a atuação do fisioterapeuta em saúde pública e a expectativa desses alunos quanto à inserção do profissional de fisioterapia no Programa de Saúde da Família (PSF). Trata-se de estudo realizado na Faculdade $M$ arechal Rondon, com 107 alunos, tendo como critério de exclusão os alunos do sétimo semestre. Foi usado um questionário, contendo onze questões, sendo oito de múltipla escol ha etrês dissertativas. O sdadospassaram por tratamento estatístico, em que foram utilizadas a análise descritiva através do programa M icrosoft Excel 2003 e análise do discurso do sujeito coletivo. A respeito da atuação do fisioterapeuta no Sistema Ú nico de Saúde (SUS), 44\% dos alunos consideram muito importante, 36\% consideraram queo papel do fisioterapeuta no PSF é muito importante, enquanto $24 \%$ dos alunos consideram não saber informar quanto à eficácia de seu atendimento. Os alunos consideram importante a atuação do fisioterapeuta no SUS e PSF, mas pouco tem conhecimento sobre a atuação do fisioterapeuta em saúde pública. Palavras-chave Fisioterapia, Saúde pública, Sistema Único de Saúde 
Introdução

História da fisioterapia no mundo

Na Antiguidade (4.000 a.C. e 395 d.C.), já havia certa ansiedade em abolir as doenças das pessoas que as apresentavam por meio de alternativas, utensílios que existiam na época como, por exemplo, o peixe elétrico como eletroterapia, usado, entre outros, como meio de terapia e tratamento de morbidades. Acredita-se que, nesta época, a ginástica estava nas mãos dos sacerdotes que a utilizavam como fins terapêuticos, ou seja, os movimentos do organismo humano, quando estudados, eram utilizados para o tratamento de doenças instaladas ${ }^{1}$.

Em 2.698 a.C., aparecem na China os primeiros registros do uso de meios físicos com o desígnio terapêutico no caso exclusivo da cinesioterapia; logo depois, na Índia. Contudo, recursos físicos naturais, como banhos de sol, são apresentados como tratamentos medicinais em toda antiguidade?

A fisioterapia surge na metade do século XIX na Europa com as primeiras escolas na Alemanha, nas cidades de Kiel, em 1902, eDresdem, em 1918. A fisioterapia surge com grande evidência no cenário mundial na Inglaterra, com os trabaIhos de massoterapia desempenhados pelos autores M endell e Cyriax, os trabalhos de cinesioterapia respiratória realizados por W inifred Linton em Londres e especialmente os trabalhos de fisi oterapia neurológica desempenhadasem conjunto com a fisioterapeuta Berta Bobath e o neurofisiologista Karel Bobath, que instituíram o método Bobath, para o tratamento de pacientes com paralisia cerebral ${ }^{2}$.

Em 1948, em Londres, foi criada a World Confederation for Physical Therapy (WCPT), que sealiou à Organização M undial da Saúde(OMS), com o intuito deativar a fisioterapia no mundo?

\section{A fisioterapia no Brasil}

O símbolo oficial da fisioterapia, um raio envolvido por uma cobra, deve-se à el etroterapia, uma modalidade terapêutica muito valorizada durante os primeiros anos da fisioterapia em nosso país².

Em 1929, na Santa Casa de M isericórdia, em São Paulo, teve início a fisioterapia no Brasil e o primeiro curso para formação de técnicos. Em 1959, foi instituída a Associação Brasileira deFisioterapeutas (ABF), que se filiou ao World Confederation for Physical Therapy (WCPT), cuja meta era procurar o auxílio técnico científico e sociocultural para a ampliação da profissão. Em 1963, o fisioterapeuta passa a ser auxiliar do médico, só podendo trabalhar sob supervisão do mesmo ${ }^{3}$.

A fisioterapia é reconhecida como curso superior em 1969 e, para legislar e estabelecer o código de ética regularizando a atuação do fisioterapeuta, criou-se o Conselho Federal de Fisioterapia e Terapia O cupacional (COFFITO) e, com a função de legalizar efiscalizar o serviço do fisioterapeuta, criou-se os Conselhos Regionais (CREFITO) conforme a Lei $n^{\circ} 6.316$ de 17 de dezembro de $1975^{3}$.

Em 1984, no Rio de Janeiro, foi criada a Associação Brasileira Beneficente de Reabilitação (ABBR), onde começa a ser ministrado o curso detécnico em reabilitação².

A Lei de Diretrizese Bases da Educação (LDB n० 9.394/96) constitui normas gerais para a educação no Brasil. No período de 1998 e 1999, COFFITO, CREFITO, coordenadores de cursos, docentes e discentes foram convidados para sugerir ao MEC as normas gerais para orientar o ensino da fisioterapia no Brasil; essas normas receberam críticas e propostas impostas pela comunidade, sendo conduzida ao Conselho Nacional de Educação. Este conselho instituiu, em 19 de fevereiro de 2002, as diretrizes curriculares nacionais do curso de graduação em fisioterapia ${ }^{3}$.

\section{Definição}

Fisioterapia é uma ciência da saúde que estuda, previne e trata os distúrbios cinéticos funcionais intercorrentes em órgãos e sistemas do corpo humano, gerados por alterações genéticas, por traumas e por doenças adquiridas. Fundamenta suas ações em mecanismos terapêuticos próprios, sistematizados pelos estudos da biologia, das ciências morfológicas, das ciências fisiológicas, das patologias, da bioquímica, da biofísica, da biomecânica, da cinesiologia, da sinergia funcional e da cinesiopatologia de órgãos e sistemas do corpo humano e as disciplinas comportamentais e sociais ${ }^{4}$.

Segundo o Consel ho Federal de Fisioterapiae Terapia Ocupacional (COFFITO), a atuação da fisioterapia pode ser em clínicas, hospitais, ambulatórios, consultórios, centros de reabilitação, em saúde coletiva, em educação eem indústria de equipamentos. Na saúde coletiva, a atuação do fisioterapeuta é em programas institucionais, ações básicas de saúde, saúde do trabalhador e 
vigilância sanitária. $\mathrm{Na}$ educação, a atuação do fisioterapeuta é em docência (níveis médio e superior), extensão, pesquisa, supervisão (técnica e administrativa), direção e coordenação de cursos eainda atua na indústria de equipamentos de uso fisioterapêutico eesporte. As especialidades reconhecidas do fisioterapeuta são a acupuntura, a quiropraxia e a osteopatia, a fisioterapia pneumofuncional, a fisioterapia neurofuncional ea fisioterapia traumatortopédica funcional ${ }^{4}$.

Quanto às exigências legais, o fisioterapeuta como pessoajurídica tem a responsabilidadetécnica pelo serviço da empresa, comprovação do registro do profissional e registro da empresa, sendo todos perante o CREFITO ${ }^{4}$.

\section{Sistema Ú nico de Saúde (SUS)}

Com as mudanças ocorridas no contexto político institucional, houve, nas últimas décadas, intensas mudanças no sistema de saúde brasileiro. Ao mesmo tempo, com o processo de redemocratização iniciado em oitenta, o país passou por séria crisenaárea econômico-financeira. Foi nesta década que buscou firmar o processo da cobertura assistencial, iniciado na segunda metade da década de setenta, atendendo as proposi ções formuladas pelas OM S na conferência de Alma-Ata em 1978, que preconizava "Saúde para todos no ano 2000 ", primordialmente por meio da atenção primária de saúde. N essa época, também, inicia o movimento da Reforma Sanitária brasileira, estabelecido inicialmente por uma pequena parte da intelectual idade universitária e dos profissionais na área da saúde. As centrais sindicais, movimentos populares da saúde e al guns parlamentares incorporaram-se posteriormente ${ }^{5}$.

As pessoas que lutam pelo Sistema Único de Saúde (SUS) reconhecem os grandes avanços do sistema ocorridos na última década, dos quais fazem parte a ampliação da extensão da população aos serviços de saúde, tanto em atenção básica de saúde como de um processo de descentralização, tornando os municípios os principais atores da saúde no sistema público ${ }^{6}$.

Foram muitas as propostas para instituir uma rede de serviços direcionada às atenções primárias à saúde, com hierarquização, descentralização e universalização, começando pelo Programa deI nteriorização das Ações de Saúdee Saneamento. O Programa Nacional de Serviços Básicos de Saúde(PREV-SAÚ DE) foi criado em 1980 mas, na realidade, não foi posto em prática. Em seguida, pelo plano do Conselho Nacional de
Administração da Saúde Previdenciária (CONASP), em 1982, foi cumprida a política de Ações Integradas de Saúde (AIS), um artifício de grande prestígio para o seguimento de descentralização da saúde?.

Os alvos principais dos sistemas da saúde são a melhoria das condições da saúde do usuário, para oferecer uma resposta apropriada às expectativas do mesmo, a adequação do tratamento e a proteção da garantia na matéria da saúde ${ }^{8}$.

Com a finalidade de se modificar a desigualdade à saúde da população, criou-se pela Constituição Federal de 1988, regulamentado pelas Leis no 8080/90 (Lei Orgânica da Saúde) e no 8.142/ 90, o SUS (Sistema Ú nico de Saúde), que torna obrigatório 0 atendimento à população gratuitamente. Dele, fazem parte os centros epostos de saúde, hospitais, incluindo os universitários, laboratórios, hemocentros, além de fundações e institutos de pesquisas, onde todos têm direito a consultas, exames einternações, nas unidades que são vinculadas aos SUS, sejam elas públicas ou privadas?.

O SUS é financiado com capital arrecadado por impostos e contribuições sociais pagos pela população e dispõe dos recursos do governo federal, estadual e municipal; tem como objetivo tornar-se um importante mecanismo de promoção à igual dade na prestação de serviços, suprindo as necessidades da saúde da população, oferecendo serviços com qualidades adequadas às situações, independente da classe social. O SUS destina-se à promoção da saúde, tendo como prioridade as ações preventivas, democratizando os esclarecimentos importantes para que a população conheça seus direitos e riscos quanto à saúde. 0 controle do acontecimento da doença, o aumento e a propagação (vigilância epidemiológica), o controle da qualidade de remédios, de exames, de alimentos e higiene, são algumas das responsabilidades de atenção do SUS. Quando as unidades públicas de assistência à saúde não são suficientes para atender à demanda de uma determinada região, se contrata o setor privado, através de convênios de prestação de serviço ao estado?.

Os níveis de atenção à saúde são classificados em primário, secundário eterciário, sendo que o primário compreende as ações voltadas para medidas preventivas, no campo da educação e da informação, junto a pessoas, grupos e a comunidade em geral. 0 nível secundário se refere às formas de identificação, diagnóstico de casos e estratégias de intervenção precoce. 0 terciário inclui 0 atendimento de serviços hospitalares e 
institucionai se comunitários ea organização dos serviços de saúde, envolvendo maior grau de complexidade, a fim de adequar a atenção aos casos de violência em termos de tratamento ou reabilitação?.

Na maioria dos países desenvolvidos, a proteção financeira em matéria da saúde pública é garantida através da ampla cobertura dos seguros públicos. Já os seguros privados tendem a funcionar como seguros substitutivos ou complementares ${ }^{10}$.

\section{O Programa de Saúde da Família}

O Programa de Saúde da família (PSF) foi criado com o objetivo de transformação da atenção primária, tendo como princípio um conjunto de atos conjuntos em harmonia com os princípios deterritorialização, intersetorialidade, descentralização, corresponsabilidade e primazia de quadro populacional com máximo perigo de adoecer ou falecer ${ }^{11}$.

A história do PSF tem princípio quando o M inistério da Saúde cria o Programa de Agentes Comunitários de Saúde(PACS), em 1991, dando início ao enfoqueà família como unidade de atividade programática enão somente o indivíduo, introduzindo a consciência deárea de cobertura. O M inistério institucionalizou nessa circunstância as vivências de atuação em saúde com agentes comunitários que já vinham se exercitando de forma solitária e acentuada em diversas regiões do país, como Paraná, Mato Grasso do Sul e Ceará. Sendo assim, pode-se assegurar que o PACS precede ao PSF, pois uma das variáveis notáveis que o PACS introduziu e que se relaciona integralmenteao PSF éque, pela primeira vez, há um enfoque interiormente às práticas de saúde. 0 programa também introduziu uma visão ativa da influência em saúde, de não esperar a demanda chegar para interferir e sim de atuar sobreela antecipadamente, compondo-se, assim, ferramenta real de reordenação da demanda ${ }^{12}$.

No processo de estabelecimento do PACS, duas questões tiveram destaque; uma foi a escoIha do agente (capacitação, avaliação, etc.) e a outra se refere às condições institucionais da administração da saúde no nível local (recursos humanos disponíveis, capacidade instalada disponível, etc.). Com isso, pode-se assegurar que, pelo episódio do PACS ter apreendido esse formato, elenão constituiu, então, em somentemais um programa reto do M inistério da Saúde ( $\mathrm{Pa}$ ralela ao Sistema de Saúde) ${ }^{12}$.
Destaca-se, todavia, que não foi somente 0 fato de ter decidido sobre a estratégia de comunicação quefez do programa um instrumento de reorganização do SUS, masigualmente o grau de articulação que esse instituiu com os diversos níveis do sistema (estadual emunicipal), além da função desempenhada pelos atores compartiIhantes do processo de execução do programa (agentes e comunidades). Esse processo se firmou em março de 1994, quando a técnica de financiamento do PACS se incluiu no pagamento por procedimentos operados pelo SUS, ou seja, a lista do Sistema de Informação Ambulatorial (SIA/SUS) ${ }^{12}$.

O PSF favorece 0 estabelecimento de novas relações, em que cada parte atuante é sujeito do processo. A tendência éque, com o tempo, o paciente deixe de ser objeto de ação, enquanto que o profissional, de qualquer instância, passe a compreendêlo enquanto ser político social, psicobiológico, cultural e contextualizado no ambiente em quevive ${ }^{13}$.

O PSF foi instituído pelo M inistério da Saúde em 1994 e o documento que estabelece as bases do programa destaca que, ao contrário do modelo tradicional, centrado na doença eno hospital, o PSF prioriza as ações de proteção e promoção à saúde dos indivíduos e da família, tanto adultos, quanto crianças, sadios ou doentes, de forma integral e contínua ${ }^{11}$.

0 princípio da citação à unidade familiar quando objeto de intervenção em saúde é anterior ao PSF. O movimento da medicina de família não se constitui numa opção crítica à teseinoportuna da educação média; além disso, a primazia da atenção primária e o reconhecimento da saúde como direito do cidadão e dever do estado requeriam posições mais realistas ${ }^{11}$.

É necessário apresentar que, a partir desse ponto, o Programa de Saúde da Família se afasta da "medicina familiar" observada. É importante ressaltar que, em primeiro lugar, o PSF se introduz no propósito da política pública de saúde, na composição do SU ${ }^{11}$. A construção de um sistema de serviços de saúde democrático universal, igualitário eintegral constitui um processo social e político que se realiza por meio de formulação de políticas públicas voltadas para a saúde e também no cotidiano dos serviços de saúde ${ }^{13}$.

Em segundo, seu conhecimento abrangeconteúdos em que se incorporam, entre outros aspectos, o modelo e a prática assistencial, o processo de atividade e a composição de recursos humanos. Trata-se de um modelo voltado para a proteção e promoção, além do atendimento 
domiciliar, com área de inclusão da clientela, equipe multiprofissional (no mínimo médico, enfermeira, auxiliar de enfermagem e agentes comunitários), atividade preventiva e de promoção à saúde, a partir deprimazia epidemiológica daárea adstrita com destaque nas ações programáticas, objetivando reduzir a demanda em centros de saúde e hospitais; e participação comunitária e controle social, fundamental nos Conselhos Municipais de Saúde. Levando-se em conta os princípios e bases operacionais do PSF, este programa pode ser bem visto como uma estratégia considerável na transfiguração do presente modelo assistencial. Todavia, é indispensável salientar que as mudanças em relação ao modelo assistencial interpretam componentes tanto técnicos quanto políticos ${ }^{11}$.

No PSF, pode-se notar que sua execução, em termos técnicos e políticos, está sendo proposta com base na interação com a comunidade, objetivando arquitetar de forma participativa práticas e artifícios mais hábeis de suportar os problemas de saúde. 0 investimento em educação continuada da equipe de saúde e em conhecimento em saúde aplicada para a população tem surtido efeitos precisos no que diz respeito à qualidade da prática assistencial e à adoção de hábitos e ações mais saudáveis por parte da população, de acordo com experiências de implantação do PSF em municípios de M inas Gerais, Ceará, Paraíba, e Pernambuco ${ }^{11}$.

O PSF repercute em componentes técnicos, políticos e comportamentais; consequentemente, uma sugestão de análise deve declinar sobre esses eixos, numa expectativa organicamente política etécnica, envolvendo aspectos quantitativos equalitativos. A agregação do atendimento domiciliar indica uma reestruturação e reorganização das práticas de saúde para além das paredes dos serviços de saúde, movendo sua visão para o espaço domiciliário das famílias e comunidades nas quais as atuações estão cravadas. Com isso, a família e sua composição tornam-se pontos estratégicos de averiguação para o $\mathrm{PSF}^{11}$.

Em Londrina, o serviço de internação hospitalar consta com a atenção em domicílio, proporcionando a recuperação rápida do paciente, diminuindo risco de infecções hospitalares e liberando leitos dos hospitais. Observou-se com isso que a atuação do fisioterapeuta em domicílio não se limita apenas em atenção primária ao paciente, mas também o contato mais próximo à família, onde mostra que a proposta do programa é de capacitar membros da família para o cuidado e auxílio com o pacienteem domicilio ${ }^{14}$.
Em Sobral (CE), o fisioterapeuta tem atuado no Programa de Saúde da Família desde 2000, no tratamento e reabilitação, com atendimentos individuais e coletivos, e propõe atividades coletivas para atendimento das crianças e adolescentes com desvios posturais e/ou com queixas de dores na coluna vertebral ${ }^{15}$.

Em um trabalho desenvolvido por Silva ${ }^{16}$, na cidade de Londrina (PR), houve comentários positivos relatados pelas mães, que mostraram, em sua maioria, satisfação na relação equipepaciente. Elas afirmaram ter sido bem tratadas e receberam atenção de toda da equipe.

\section{A atual situação da fisioterapia nas Unidades Básicas deSaúde}

Os problemas da saúde da população são fundamentais no processo do planejamento sanitário. Não obstante, a maneira em queos problemas de saúde são percebidos pode ter sentidos diversos. A percepção dos usuários não é frequentemente estritamente médica, porque para estes a falta do transporte, da discriminação social e da qualidade das relações humanas entre o pessoal da saúde e os pacientes podem determinar fatores na percepção dos problemas sanitários ${ }^{17}$.

O fisioterapeuta pode atuar em todos os níveis de atenção à saúde, sendo estes primário, secundário eterciário, dentro da equipe interdisciplinar. Contudo, em função de aspectos de ordem político-econômica e organizacionais, sua ocupação é pouco transmitida e subutilizada. Todavia, vagarosamente, demonstrações isoladas em algumas regiões brasileiras mostram que a inserção da fisioterapia no Programa de Saúde da Família abrilhanta ainda mais a atenção de saúde da população ${ }^{18}$.

A atuação do fisioterapeuta não se limita apenas ao setor curativo e de reabilitação; as ações de prevenção e educação em saúde são essenciais para a melhora da qualidade de vida da população e as intervenções no atendimento domiciliar podem levar à positividade na relação do paciente com o meio, tanto físico quanto social ${ }^{19}$.

A fisioterapia no PSF de Sobral (CE) está distribuída em grandes e pequenas áreas, cada uma com um nível de abordagem encaminhado, aderido às diversas patologias e condições desdeneurológicas a ginecobstétricas ${ }^{18}$.

0 potencial é mostrado pelos trabalhos que os fisioterapeutas vêm cumprindo no Programa de Saúde da Família, instituídos na Unidade Básica de Saúde (UBS) e incluídos nas mais diver- 
sas atividades: grupos de gestantes, grupos de postura, grupos de mãe de crianças com infecção respiratória aguda, grupo de prevenção de inaptidão em hanseníase, grupo de mães com filhos com problemas neurológicos, grupo de idosos, proceder na saúde da criança, atendimento individual, estimulação necessária em crianças com atraso no desenvolvimento neuropsicomotor, trabalho nas creches, reeducação postural global, restabelecer cuidadores dentro do ambiente familiar de orientação à saúde, trabaIhadores vítimas de acidentes de trabalho e de doenças do trabalho (lesões por esforços repetitivos - LER/doença osteomuscular relacionada ao trabalho - DORT), crianças em idade escolar sob riscos ergonômicos das escolas, diabéticos e hipertensos, pacientes acamados e usuários de prótese e órtese ${ }^{19}$.

Também conforme Ferreir $\mathrm{a}^{20}$, a fisioterapia pode proceder junto à equipe, realizando atendimentos domiciliários em pacientes acamados ou impossibilitados, realizando atividades para que consigam desenvolver suas atividades de vida diária (AVD), melhorando a qualidade de vida e evitando possíveis complicações. A participação da fisioterapia na saúde coletiva compõe-se em uma contribuição imperativa que pode viabilizar maior decisão junto a outros profissionais.

Ao longo dos três anos de graduação, mesmo com a disciplina de saúde coletiva na estrutura curricular do primeiro ano, percebe-se 0 pouco conhecimento por parte dos alunos em relação à atuação do fisioterapeuta em saúde coletiva. O levantamento de dados da pesquisa teve como base verificar o conhecimento dos alunos em relação ao profissional de fisioterapia. Este trabalho poderá servir de referência para novas pesquisas sobre a diversidade do campo de trabalho da fisioterapia em saúde coletiva.

\section{Objetivos}

O objetivo geral deste estudo foi verificar a expectativa dosalunos de primeiro, terceiro equinto semestres do curso de fisioterapia sobre a atuação da fisioterapia em saúde pública ea expectativa dos mesmos alunos na inserção deste profissional na equipe do Programa de Saúde da Família (PSF). Dentre os objetivos específicos, saber o nível de conhecimento dos alunos sobre as diferentes formas de atuação da fisioterapia em saúde coletiva; conhecer a expectativa dos alu- nos sobre a atuação do fisioterapeuta em saúde coletiva e efetuar comparações entre dados obtidos a partir do questionário aplicado com a re visão de literatura acerca da atuação do fisioterapeuta em saúde coletiva.

\section{Materiais emétodos}

Este estudo foi realizado na Faculdade M arechal Rondon (FM R), situada na cidade de São Manuel (SP), na qual foi aplicado um questionário a 107 de 183 alunos regularmente matriculados, de ambos os sexos, com idade média de 24 anos.

Trata-se de um estudo transversal, com uma abordagem quantitativa e qualitativa.

Para a coleta e organização das informações de interesse, não foi encontrado na literatura um questionário que correspondesse a essa pesquisa. Foi elaborado um questionário contendo onze questões, sendo elas oito de múltipla escolha, no qual da primeira a quarta questão continham as alternativas: nenhuma importância, muito pouco importante, pouco importante, importante, muito importante, mais que importante e não sei informar. Da quinta à oitava questão, as alternativas eram: ruim, regular, bom, muito bom, ótimo, excelente e não sei informar. As questões 9 e 10 eram questões objetivas numéricas ea questão 11 , dissertativa.

0 projeto de pesquisa foi submetido e aprovado pelo Comitê de Ética e Bioética (COEBE) da FMR.

\section{Recrutamento dos alunos}

Para participação da pesquisa, os alunos foram recrutados em sala de aula com convite verbal. 0 questionário foi aplicado em sala de aula com a autorização do professor responsável, os alunos foram devidamente esclarecidos sobre a proposta do trabalho, sobre o teor do questionário, assinaram o termo de consentimento livre e esclarecido e tiveram tempo livre para responder as questões.

Foram distribuídos os questionários aos alunos, sendo que todos os questionários aplicados foram devidamente respondidos, não tendo a de volução de nenhum questionário sem respostas.

Não havia no questionário a especificação do sexo feminino e masculino e sabe-se através da secretaria da FMR que há predomínio do sexo feminino no curso de fisioterapia. 


\section{Critérios de inclusão}

Participaram da pesquisa todos os alunos do primeiro, terceiro equinto semestres do curso de fisioterapia, presentes em sala de aula na data da aplicação do questionário, sendo $90 \%$ com idade entre dezoito e trinta anos, conforme dados cedidos pela secretaria da instituição de ensino.

\section{Critérios de exclusão}

Foram excluídos da pesquisa os alunos do sétimo semestre de fisioterapia por já estarem participando dos estágios supervisionados, incluindo o módulo de fisioterapia em saúde coletiva, o que poderia causar um viés nos resultados.

Tratamento dos dados

Para a avaliação estatística dos dados coletados da primeira à oitava questão, foi utilizada a análise descritiva através do programa Microsoft Excel 2003 por meio de média aritmética. Da nona à décima primeira questão, realizou-seanálise do discurso do sujeito coletivo (Lefèvre), por meio de frequência de respostas.

\section{Resultados}

D os 107 alunos entrevistados de 183 regularmente matriculados com média de idade de 24 anos de ambos os sexos do primeiro, terceiro equinto semestres do curso de fisioterapia, obtivemos os seguintes resultados:

Dos alunos analisados, 44\% consideram muito importante o papel do fisioterapeuta no SU S enquanto 3\% não souberam informar (Gráfico 1).

Para 37\% dos alunos, o papel do fisioterapeuta no PSF é muito importante, enquanto $1 \%$ considerou pouco importante (Gráfico 2).

Dos entrevistados, $69 \%$ dos alunos consideraram mais que importante a importância da preparação do fisioterapeuta para 0 atendimento da rede pública, enquanto $1 \%$ considerou nenhuma importância (Gráfico 3).

Dos al unos entrevistados, $43 \%$ consideraram muito importante a qualificação/especialização do fisioterapeuta na rede pública, enquanto $1 \%$ considerou nenhuma importância (Gráfico 4).

Em relação à qualidade do atendimento da fisioterapia na rede pública, $25 \%$ dos al unos consideram não saber informar, enquanto $6 \%$ consideraram ruim (Gráfico 5).
Em relação à eficácia do atendimento da fisioterapia na rede pública, $24 \%$ dos alunos consideram não saber informar, enquanto $4 \%$ consideram ruim.

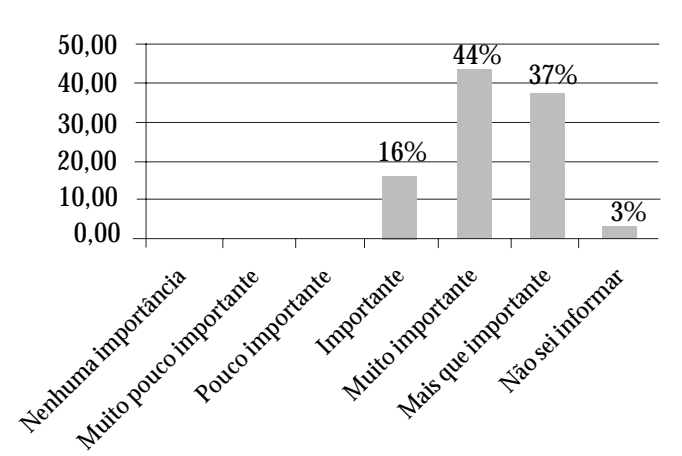

Gráfico 1. Papel do fisioterapeuta no SUS, 2006.

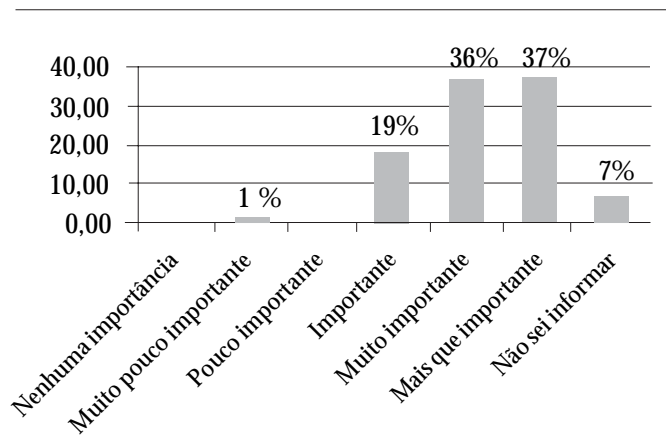

Gráfico 2. Papel do fisioterapeuta no PSF, 2006.

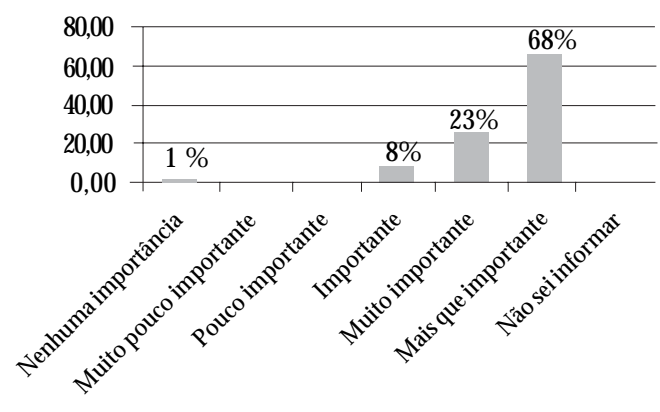

Gráfico 3. Importância da preparação do fisioterapeuta para 0 atendimento da rede pública, 2006. 


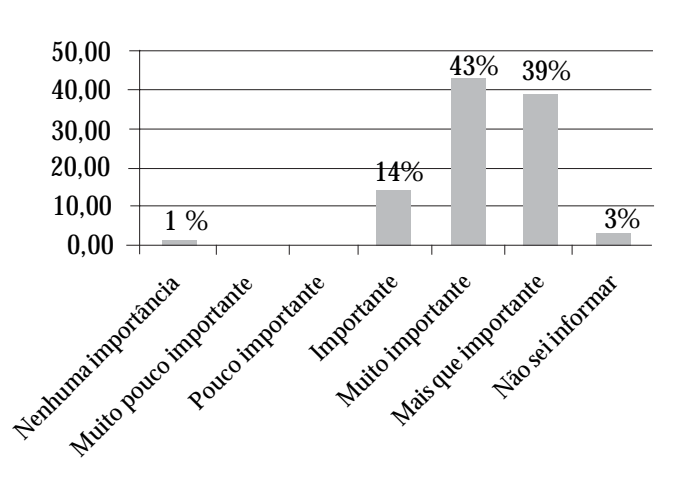

Gráfico 4. Qualificação/especialização do fisioterapeuta na rede pública, 2006.

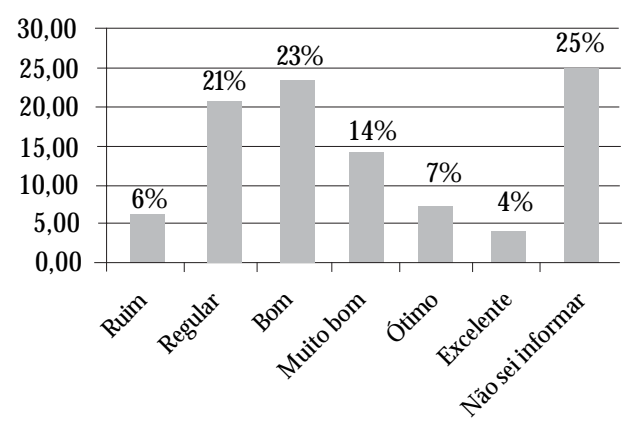

Gráfico 5. Qualidade do atendimento da fisioterapia na rede pública, 2006.

Dos alunos entrevistados, $22 \%$ consideram muito bom a jornada de trinta horas semanais, enquanto $3 \%$ consideram ruim.

Em relação ao piso salarial de $R \$ 1.285,00$ por trinta horas semanais, $31 \%$ dos alunos afirmam não saber informar, enquanto $2 \%$ consideram excelente.

Dos alunos entrevistados, $61,68 \%$ ou 66 alunos acham que o número de atendimentos individuais que o fisi oterapeuta deve realizar por seis horas diárias é entre cinco e seis atendimentos, enquanto onzealunos ou $10,28 \%$ consideram que devem ser realizados entre dez e doze atendimentos (Tabela 1).

Em relação ao número máximo de pacientes por fisioterapeuta para atendimento em grupo $52,34 \%$ ou catorzealunos acham que éentreseis, cinco etrês pacientes, enquanto $18,69 \%$ ou vinte alunos não souberam informar (Tabela 2).

Independente do número de alunos em cada resposta, todos mostraram conhecimento da grande variedade na atuação do fisioterapeuta em Unidade Básica de Saúde (Tabela 3).

\section{Discussão}

Verificou-se que os alunos consideram importante a atuação do fisioterapeuta no SUS-PSF, assi $m$ como a preparação equalificação do atendimento em saúde coletiva. Ferreira et al. ${ }^{20}$ define em seu estudo que a participação da fisioterapia na saúde coletiva estabelece-se em uma cooperação imperativa, que pode viabilizar maior decisão em conjunto com outros profissionais.

No estudo feito por Arruda ${ }^{21}$, a autora ressalta ser muito importante tornar a fisioterapia acessível à população. É crescente o número de pessoas nas comunidades que necessitam deste serviço e não possuem condições de deslocar-se para a Unidade Básica de Saúde.

Segundo Ribeiro, citado por Ragasson ${ }^{22}$, a inserção do fisioterapeuta nos serviços de atenção primária à saúde é um processo em construção, em associação à criação da profissão, intitulando o fisioterapeuta como reabilitador, voltando apenas para uma pequena parte do seu objetivo de trabalho, que étratar as doenças e suas sequelas. Essa lógica de conceitualização durantemuito tempo exclui da rede básica os serviços da fisioterapia, levando a uma grande dificuldade da população em ter acesso a esse serviço, impedindo o profissional de atuar na atenção primária.

Os alunos pouco têm conhecimento sobre a atuação da fisioterapia em saúde pública, pois ainda é escasso o número de profissionais nesta área, visto que o profissional de fisioterapia ainda não faz parte da equipe multidisciplinar. Cabem mais pesquisas na área, buscando relacionar o conhecimento da atuação da fisioterapia em saúde coletiva e a utilização por parte dos alunos de graduação em instituições de nível superior do Sistema Ú nico de Saúde. A pouca quantidade de fisioterapeuta no PSF é questionada, pois falta aos conselhos e associações profissionais destas áreas uma proposta do que pode ser feito na organização desses serviços nos municípios, dentro de uma visão de saúde pública. Seria de suma importância sob o ponto de vista crítico a inserção profissional do fisioterapeuta no PSF, visto que ele pode atuar de maneira preventiva $e$ no tratamento nas diversas enfermidades ${ }^{14}$.

Os alunos questionados mostram-se preocupados em garantir boa qualidade no atendimento.

Ferreira et al. ${ }^{20}$ concluem em sua pesquisa que a presença do fisioterapeuta na comunidade se 
torna fundamental na medida em que contribui para a promoção, prevenção, recuperação e reabilitação, obedecendo assim aos princípios do atual modelo desaúdee, consequentemente, promovendo a melhoria da qualidade de vida da população ${ }^{20}$.

Ragasson ${ }^{22}$ constatou em sua pesquisa que a fisioterapia apresenta papel importante, de cooperação, mediante a nova realidade de saúde que se apresenta por meios terapêuticos físicos, na prevenção, eliminação ou melhora da qualidade de vida, na promoção e na educação em saúde. 0 atendimento domiciliar é fundamental ao trabaIho de atenção primária do profissional fisiotera-

Tabela 1. Número de atendimentos individuais que o fisioterapeuta deve realizar por seis horas diárias, 2006.

\begin{tabular}{lrr}
\hline $\begin{array}{l}\text { Atendimentos individuais } \\
\text { por seis horas diárias }\end{array}$ & $\mathrm{n} \cong$ & $\%$ \\
\hline 09 atendimentos & 1 & 0,93 \\
04 atendimentos & 2 & 1,87 \\
07 atendimentos & 2 & 1,87 \\
08 atendimentos & 3 & 2,80 \\
12 atendimentos & 5 & 4,67 \\
10 atendimentos & 6 & 5,61 \\
Não soube informar & 10 & 9,35 \\
Outros & 12 & 11,21 \\
05 atendimentos & 14 & 13,08 \\
06 atendimentos & 52 & 48,60 \\
Total & 107 & 100,00 \\
\hline
\end{tabular}

Tabela 2. Número máximo de pacientes por fisioterapeuta, para atendimento em grupo, 2006.

\begin{tabular}{lrr}
\hline \multicolumn{1}{c}{ Pacientes em grupo } & $\mathrm{n} \cong$ & $\%$ \\
\hline 07 pacientes & 1 & 0,93 \\
25 pacientes & 1 & 0,93 \\
01 pacientes & 2 & 1,87 \\
08 pacientes & 2 & 1,87 \\
12 pacientes & 2 & 1,87 \\
15 pacientes & 2 & 1,87 \\
20 pacientes & 3 & 2,80 \\
10 pacientes & 5 & 4,67 \\
Outros & 5 & 4,67 \\
02 pacientes & 6 & 5,61 \\
04 pacientes & 11 & 10,28 \\
06 pacientes & 11 & 10,28 \\
05 pacientes & 17 & 15,89 \\
03 pacientes & 19 & 17,76 \\
Não soube informar & 20 & 18,69 \\
Total & 107 & 100,00 \\
\hline
\end{tabular}

peuta, pois nos deparamos com a realidade das pessoas, constatando suas atividades de vida diária, suas limitaçõese, a partir disso, direcionamos os encaminhamentos e orientações a cada caso.

Segundo fontes do M inistério da Saúde de 2006, é de extrema importância o compromisso nos esforços de qualificação do processo de gestão, aprimorando o rendimento e a efetividade da administração pública, para que se consiga implementar políticas que impactem positivamente o perfil da saúde e a qualidade de vida das populações, não deixando de ressaltar a complexidade da responsabilidade de governar no mundo contemporâneo, global e de poderes associados, em que a realidade se apresenta de várias formas e mais dinâmica, assim como as especificidades do setor saúde, no que se refere aos processos de decisão, programação, execução e avaliação das ações ${ }^{23}$.

\section{Considerações finais}

Pela análise real izada, pode-severificar queosalunos consideram importante a atuação do fisioterapeuta no SUS-PSF, assim como a preparação e qualificação do atendimento na rede pública.

Os alunos pouco têm conhecimento sobre a atuação da fisioterapia em saúde pública, pois ainda é escasso o número de profissionais nesta área, visto que o profissional de fisioterapia ainda não faz parte da equipe multidisciplinar. Cabem mais pesquisas na área, buscando relacio-

Tabela 3. Atuação do fisioterapeuta em uma Unidade Básica de Saúde/Posto de Saúde, 2006.

\begin{tabular}{lrr}
\hline \multicolumn{1}{c}{$\begin{array}{c}\text { Atuação do fisioterapeuta } \\
\text { em Unidade Básica de Saúde }\end{array}$} & $\mathrm{n} \cong$ & $\%$ \\
\hline Assistência a acamados & 1 & 0,59 \\
Atendimento em grupo & 1 & 0,59 \\
Atuar em atenção secundária & 1 & 0,59 \\
Campanhas & 1 & 0,59 \\
Conforme a necessidade & 1 & 0,59 \\
Desconhece tal atendimento & 1 & 0,59 \\
Atenção básica à saúde & 2 & 1,18 \\
Palestras educativas & 2 & 1,18 \\
Atuar em nível primário & 3 & 1,78 \\
Conscientização & 5 & 2,96 \\
Orientação & 13 & 7,69 \\
Promoção à saúde & 23 & 13,61 \\
Prevenção & 35 & 20,71 \\
Reabilitação & 35 & 20,71 \\
Não soube informar & 45 & 26,63 \\
Total & 169 & 100,00 \\
\hline
\end{tabular}


nar o conhecimento da atuação da fisioterapia em saúde coletiva e a utilização por parte dos alunos de graduação em instituições de nível superior do Sistema Ú nico de Saúde. Os alunos se preocupam em garantir boa qualidade no atendimento dos pacientes.

\section{Referências}

1. Rebelatto JR, Botomé SP. As alterações na concepção do objeto de trabalho em Fisioterapia em diferentes momentos de sua constituição. In: Rebelatto $J R$, Botomé SP, organizadores. Fisioterapia no Brasil. 2a ed. São Paulo: Manole; 1999. p. 31-32.

2. Gava MV. Retrospectiva da formação do fisioterapeuta no Brasil. In: Gava M V, organizador. Fisioterapia: História, reflexões e perspectivas. 1a ed. São Bernardo: M etodista; 2004. p. 27-30.

3. Universidade de Ribeirão Preto. Histórico da Fisioterapia no Brasil. [site da Internet]. [acessado 2006 out 06]. Disponível em: http://fisioterapia.unaerp.br

4. Conselho Federal de Fisioterapia e Terapia Ocupacional. Definição de Fisioterapia. [site da Internet]. [acessado 2006 mai 15]. Disponível em: http://www. coffito.org.br

5. Brasil. M inistério da saúde. [site da Internet] [acessado 2006 nov 12]. Disponível em: http://portal. saude.gov.br

6. Barata LRB, Tanaka OU, M endes JDV. 15 anos do SUS: desafios e perspectivas. Saúde em revista 2003; 5(11):7-14.

7. Brasil. Ministério da Saúde. Sistema Único de Saúde. [site da Internet] [acessado 2006 ago 18]. Disponível em: http://www.sespa.pa.gov.br

8. Puentes ER, Ruelas E, Martínes TM, Garrido FL. Trato adecuado: respuesta del sistema mexicano de salud a las expectativas no médicas de sus usuários. Salud pública de M éxico 2005; 47(Supl. 1):12-21.

9. Gomes R, Silva CM FP, Njaine K. Prevenção à violência contra a criança e o adolescente sob a ótica da saúde: um estudo bibliográfico. Cien Saude Colet 1999; 4(1):171-181.

10. Rosas EP, Sesma S, Dantés G. Estimación de la población com seguro de salud en Mexico mediante una encuesta nacional. Salud pública de M exico 2005; 47(Supl. 1):22-26.

11. Trad LAB, Bastos ACS. O Impacto sócio- cultural do Programa de Saúde da Família (PSF): uma proposta de avaliação. Cad Saude Publica 1998; 14(2):429-435.

12. Viana ALD, Poz MRD. A reforma do sistema de saúde no Brasil e o Programa de Saúde da Família. Revista de Saúde Coletiva 2005; 15(Supl.):225-264.

13. Santana ML, Carmagnani MI. Programa saúde da Família no Brasil: Um enfoque sobre seus pressupostos básicos, operacionalização e vantagens. Saude soc. 2005; 10(1):1-25.

\section{Colaboradores}

CR N aves eVS Brick participaram igualmente de todas as etapas da elaboração do artigo.

14. Felicio DNL, Franco ALV, Torquato MEA, Abdon APV. Atuação do Fisioterapeuta no atendimento domiciliar de pacientes neurológicos: A efetividade sob a visão do cuidador. RBPS 2005; 18(2):64-69.

15. Monteiro M PA. Atendimento Coletivo às Crianças com Problemas na Coluna Vertebral. [site da Internet] [acessado 2006 jun 01]. Disponível em: http:// www.sobral.ce.gov.br/saudedafamilia/publicacoes/ abrasco

16. Silva AM R, Andrade SM, Thomson Z. O piniões de mães de crianças que morreram no primeiro ano de vida no M unicípio de Londrina, Paraná, Brasil. Cad Saude Publica 2002; 18(5): 1295-1302.

17. Muñoz AMA, Herrera ACV. Dimensiones culturales en el proceso de atención primaria infantil: perspectivas de las madres. Salud pública de M exico 2005; 47(6):440-446.

18. Brasil ACO, Brandão JAM, Silva MON, Godim FiIho VC. O papel do Fisioterapeuta do Programa Saúde da Família do M unicípio de Sobral-Ceará. RBPS 2005; 18(1):3-6.

19. Maciel RV, Silva PTG, Sampaio RF, Drummond AF. Teoria, prática e realidade social: uma perspectiva integrada para 0 ensino de fisioterapia. Fisioterapia em M ovimento 2005; 18(1):11- 17.

20. Ferreira FN, Leão I, Saqueto $M B$, Fernandes $M H$. Intervenção fisioterapêutica na comunidade: relato de caso de uma paciente com AVE. Rev. Saúde.com 2005; $1(1): 35-43$

21. Arruda AD, Guedes BN, Lima FR, Ribeiro KSQS, Cavalcanti RLL. A importância da inclusão da fisioterapia no programa saúde da família. [site da Internet]. [acessado 2006 nov 04]. Disponível em: http:/ /www.prac.ufpb.br

22. Ragasson CAP, Almeida DCS, Comparin K, Mischiati M F, Gomes JT. Atribuiç̃es do fisioterapeuta no programa de saúde da família: reflexões a partir da prática profissional. [site da Internet]1998 [acessado 2006 nov 04 ]. Disponível em: http://www.unioeste.br/ projetos/saudefamili a/atribuições_psf.rtf

23. Brasil. M inistério da Saúde. Informação, ferramenta fundamental a boa gestão. [site da Internet] [acessado 2006 nov 05]. Disponível em: http://portal.saude. gov.br/portal/saude/area.cfm?id_area=997

Artigo apresentado em 22/07/2008

Aprovado em 29/01/2009

Versão final apresentada em 22/02/2009 